\title{
STUDIES ON SERO PREVALENCE OF HEPATITIS B AND C AMONG HIV PATIENTS ATTENDING SOME HOSPITALS IN KANO
}

\author{
Bashir, M. ${ }^{1}$ and Aliya, S.M. ${ }^{2}$ \\ Department of Biological Sciences, Bayero University, P.M.B. 3011, Kano, Nigeria \\ Correspondence author: bshchemiron@yahoo.com
}

\begin{abstract}
Two hundred Human Immunodeficiency Virus (HIV) infected patients, i.e. one hundred from Infectious Diseases Hospital (IDH) and the other hundred from Sir Muhammad Sanusi Specialist Hospital (SMSSH) Kano were screened for Hepatitis B and C surface antigens (HBSAg and HCSAg), from July - September, 2008 using Rapid Test Strip. The result of the rapid diagnostic test among the patients attending IDH, indicated that 14 (14\%) of the subjects were seropositive for HBSAg, with eleven (25.6\%) been males, while 3(5.3\%) were females. Similarly at SMSSH, eighteen (18\%) of the subjects screened were positive for HBSAg, out of which males were having higher number of co - infection 11 (34.4\%), while the females were $7(10.3 \%)$. The entire subjects tested in the two hospitals were seronegative for HCSAg. Furthermore, patients in the age group of 31 - 40 were found to have the highest rate of infection $23.1 \%$ at SMSSH and $22 \%$ at IDH respectively. Statistical analysis of the results had demonstrated that there was significant difference between infected males and infected females. In conclusion, there was high rate of HBSAg among HIV infected patients in Kano, while hepatitis C viral infection was rare among HIV patients in Kano.
\end{abstract}

Keywords; HIV, HBSAg, HCSAg, IDH, SMSSH, Rapid Diagnostic test.

\section{INTRODUCTION}

Inflammation of the liver (Hepatitis) could be caused by a number of etiological agents such as viruses, bacteria, fungi, drugs, and other chemicals. The situation is characterized by distortion of the normal hepatic lobular architecture due to varying degree of growth of liver cells (Zeore and Walter, 1990). The term viral hepatitis refers to only those cases of liver disease caused by hepatitis viruses labeled from A $G$, hepatitis virus $A, E$ and $F$ are largely transmitted via ingestion of contaminated food or water other wise called faecal - oral route, while B,C, D, and G viruses are mainly transmitted by blood or bodily fluids (Cheesbrough, 2004). Regardless of the type of hepatitis, patients with viral hepatitis may experience low grade fever, headache, muscle ache, vomiting, abdominal pain, joints pain, dark coloured urine, pale bowel movement, pain in the upper right quadrant of the abdominal cavity and jaundice. Hepatitis B and C viral infections are common among HIV infected patients because of the shared routes of viral transmission and it is becoming a great public health concern world wide Chen et al., 2011. The sub Saharan Africa has been most severely affected by the HIV/AIDS pandemic with almost $9 \%$ of its adult population living with HIV (WHO, 2002). The HIV/AIDS epidemic in Nigeria has extended beyond the commonly classified high - risk groups and is now common in the general population with the adult prevalence rate of $5.8 \%$ in 2001 (FMH, 2003). Hepatitis $B$ virus is the most common cause of serious liver infection in the world, it is estimated that more than two billion people have been infected by HBV world wide and 350 million people have chronic infection (Drosten et al., 2004). The virus is highly contagious and relatively easy to transmit from one infected individual to another by blood contact, mother to child during birth, unprotected sex and by sharing of needles (Finlayson et al., 1999). Nigeria is classified among the group of countries highly endemic for HBV infection with $75 \%$ of the population at risk having been exposed to the virus at one time or the other. Hepatitis B virus infection increases the risk of liver cirrhosis, and death from liver disease especially in patients with low $\mathrm{CD}_{4}^{+}$cell count as in HIV infection (Thio et al., 2002). Hepatitis C virus is a life threatening viral infection of the liver. Approximately 170 million people world wide are chronically infected with the virus and the infection is described as silent because people may be infected for 10 - 30 years with no exhibit symptoms (Seef, 1998). Co - infection of HCV and HIV is quite common due to the shared route of viral transmission and most studies have shown that HIV infections leads to a more aggressive $\mathrm{HCV}$ infection and a higher risk of liver damage (Taura, 2007). The natural history and progression of $\mathrm{HCV}$ infection is accelerated in patients with HIV, leading to increased rate of progression to cirrhosis, decompensated liver disease, hepato cellular carcinoma and death (Pineda et al., 2005; Merrchante et al., 2006). In the United States (US), HIV and HCV Co - infection is most prevalent among patients who have history of either haemophilia or intravenous drug use and among these patients, the rate of co infection could reach $70-95 \%$ as compared to $1-$ $12 \%$ among homo sexual men (Alter, 2006). The research was aimed at determining the prevalence of the hepatitis B and C viral infections among HIV infected subjects. Similarly, to determine the rate at which the two hepatitis viral infectious co - exist in HIV infected subjects. 


\section{MATERIALS AND METHODS \\ Sampling Sites}

The sampling sites were Infectious Diseases Hospital in Fagge Local Government and Sir Muhammad Sanusi Specialist Hospital, Nassarawa Local Government, Kano.

\section{Sample Size;}

The sample size was calculated using the prevalence rate of HIV in Nigeria to be $4.6 \%$ as reported by $\mathrm{FMH}$ (2008). A standard epidemiological formula was used to calculate the sample size as follows;

$$
\begin{aligned}
& n=\frac{Z^{2} P q}{d^{2}} \\
& n=\frac{1.9^{2} \times 0.046 \times(1-0.046)}{0.05^{2}}=\frac{0.177 \times 0.954}{0.0025}=67
\end{aligned}
$$

$\mathrm{n}=$ Sample size, $\mathrm{z}=$ standard normal distribution at 95 confidence interval, $p=$ prevalence rate $4.6(0.046)$, $q=1-p, 1=$ is the maximum value of probability, $d=$ allowable error taken as 5\% (0.05)

\section{Sample Collection}

With permission from the Kano State Hospital Management Board, $5 \mathrm{~mL}$ of venous blood were aseptically collected into anticoagulant free disposable plastic sample bottle using disposable plastic syringe and needle (BD) from 200 HIV infected patients attending the two hospitals. The sample size was increased from 67 to 200 in order to obtain reliable prevalence rates. Each container was then labeled and centrifuged at $2000 \mathrm{rpm}$ for five minutes. The sera were separated from the sediments and immediately tested for Hepatitis B and C surface antigens. Rapid strips for HBSAg and HCSAg were removed from the sealed pouches, with the arrows of the strips pointing toward the serum; each strip was immersed vertically in the serum for 15 seconds avoiding the serum level passing the maximum line on the strips as described by the Manufacturers. The strips were then placed on a non absorbent surface and the liner was started. The results obtained were interpreted at 15 minutes. Positive samples were not noted with two distinct coloured red lines appearing on both the test and control region with their intensity varying depending on the concentration of the HBSAg or HCSAg present in the sample. Negative samples were noted with only one coloured line appearing in the control region and no apparent red or pink line on the test region. The specificity and sensitivity of the test strips were 99.3 and 100 respectively as reported by the Manufacturers. Information about the age of the subjects was obtained through verbal interview with them.

\section{RESULTS AND DISCUSSION}

Fourty three male patients and fifty seven female subjects were investigated in IDH during the period of the study. Eleven $(25.6 \%)$ of the male subjects were positive pf HBSAg, while $3(5.3 \%)$ of the female patients were positive for HBSAg, the overall incidence was 14 (Table 1). All the patients investigated were negative for HCSAg.

The highest rate of HBSAg were recorded among the age group $31-40(9,22 \%)$, then $21-30$ years $(3,7.5 \%)$, however, co - infection was not observed among HIV infected patients of less than 20 years and 51 - 70 years respectively (Table 2 ).

Thirty two male subjects and sixty eight female subjects were investigated at SMSSH during the study period, $11(34.4 \%)$ of the male patients were found to be positive for HBSAg, while only $7(10.3 \%)$ of the female subjects were found to be positive, the overall incidence was 18 . However, all the subjects investigated were found to be negative for HCSAg (Table 3).

The highest rate of co - infection was found among $41-50(45.5 \%)$ and 31 - 40 age groups (23.1\%), while 21 - 30 age group has five co - infected individuals $(11.1 \%)$, however, less than 20 years also recorded $(11.1 \%)$ rate of co - infection (Table 4 ).

Table 1: Sero Prevalence of HBSAg and HCSAg among Hiv patients at IDH

\begin{tabular}{cccccc}
\hline Sex & No of HIVPositive & No of HBSAg Positive & $\mathbf{\%}$ & No of HCSAg & $\mathbf{\%}$ \\
\hline Male & 43 & 11 & 25.6 & 0 & 0 \\
Female & 57 & 3 & 5.3 & 0 & 0 \\
Total & 100 & 14 & 14 & 0 & 0 \\
Key; HBSAg= Hepatitis & B Surface & Antigens HCSAg & $=$ Hepatitis C Surface Antigen HIV= - Human
\end{tabular}

Immunodeficiency Virus,

Table 2: Age related Sero Prevalence of HBSAg and HCSAg at IDH

\begin{tabular}{llllll}
\hline Age Grp. & HIV Positive & HBSAg positive & \% & No of $\mathbf{H C S A g}$ & $\mathbf{\%}$ \\
\hline$<20$ & 8 & 0 & 0 & 0 & 0 \\
$21-30$ & 40 & 3 & 7.5 & 0 & 0 \\
$31-40$ & 41 & 9 & 22 & 0 & 0 \\
$41-50$ & 8 & 2 & 2.5 & 0 & 0 \\
$51-60$ & 2 & 0 & 0 & 0 & 0 \\
$61-70$ & 1 & 0 & 0 & 0 & 0 \\
Total & 100 & 14 & 14 & 0 & 0 \\
\hline
\end{tabular}


Table 3: Sero Prevalence of HBSAg and HCSAg co-infection among HIV patients at SMSSH

\begin{tabular}{llrlrl}
\hline Sex & HIV Positive & No of HBSAg & $\%$ & No of HCSAg & \% \\
\hline Male & 32 & 11 & 11 & 0 & 0 \\
Female & 68 & 7 & 7 & 0 & 0 \\
Total & 100 & 18 & 18 & 0 & 0 \\
\hline
\end{tabular}

Table 4: Age related Sero Prevalence of HBSAg and HCSAg among HIV patients at SMSSH

\begin{tabular}{llrrrr}
\hline Age Group & HIV Positive & No of HBSAg & $\%$ & No of HCSAg & $\%$ \\
\hline$<20$ & 18 & 2 & 11.1 & 0 & 0 \\
$21-30$ & 44 & 5 & 11.4 & 0 & 0 \\
$31-40$ & 26 & 6 & 23.1 & 0 & 0 \\
$41-50$ & 11 & 5 & 45.5 & 0 & 0 \\
$51-60$ & 1 & 0 & 0 & 0 & 0 \\
Total & 100 & 18 & 18 & 0 & 0 \\
\hline
\end{tabular}

Table 5: Age related Sero Prevalence of HBSAg and HCSAg among HIV patients at SMSSH

\begin{tabular}{llclll}
$\begin{array}{l}\text { Class } \\
\text { Interval }\end{array}$ & HIV & No of HBV & \% & No of HCV & \% \\
\hline$<20$ & 8 & 0 & 0 & 0 & 0 \\
$21-30$ & 40 & 3 & 7.5 & 0 & 0 \\
$31-40$ & 41 & 9 & 22 & 0 & 0 \\
$41-50$ & 8 & 2 & 2.5 & 0 & 0 \\
$51-60$ & 2 & 0 & 0 & 0 & 0 \\
$61-70$ & 1 & 0 & 0 & 0 & 0 \\
Total & 100 & 14 & 14 & 0 & 0
\end{tabular}

Table 6. Overall sero-prevalence of HIV and HBSAg between male and female patients in IDH and SMSH Kano

\begin{tabular}{lccc}
\hline Sex of patients & no. of patients examined & no. Positive & Percent \\
\hline Male & 75 & 22 & 29.3 \\
Female & 125 & 10 & 8.0 \\
Total & 200 & 32 & \\
$X^{2}$ value 18.71 & & & \\
$X^{2}(1 \mathrm{df}, 0.05,3.841)$ & & & \\
\hline
\end{tabular}

\section{DISCUSSION}

The fourteen percent sero prevalence of Hepatitis $B$ surface Antigen (HBSAg) among Human Immunodeficiency Virus (HIV) infected patients at Infectious Disease Hospital (IDH) and 18\% among Human Immunodeficiency Virus (HIV) infected patients at Sir Muhammadu Sanusi Specialist Hospital (SMSSH) could indicate that Kano State is highly endemic for hepatitis B viral infection among its HIV patients. This could be related to the classification of high endemicity of HBV as HBSAg greater than $7 \%$ in an adult population as revealed by Hodges et al. (1998). This finding was in conformity with the result obtained from studies conducted in some parts of Nigeria where the prevalence of HBSAg was found to be between 7.4 - 26\% as reported by Ekpo et al. (1995). Similarly, sex related sero prevalence of HBSAg analysis has shown that infection among males were higher than females at both study sites. This may be due to the multiple sexual partnership and promiscuity which are common habits occurring with high frequency among the male than the females. Similarly, the high rate of infection by males could be related to the fact that they are more expose to road accidents, violent events etc which increase the possibility of HBV transmission. Furthermore, aged related sero prevalence of $\mathrm{HBSAg}$ analysis have demonstrated that age group $31-40$ years had the highest prevalence rate at both study sites, which could be associated with multiple sexual partnerships reported to be highest among individuals in their third decades of life. Moreover, among the HIV infected subjects the higher the rate of HBSAg could be related to the decline in the body's defense mechanism due to the destruction of $\mathrm{CD}_{4}{ }^{+}$lymphocytes by the virus. In addition, HCV was not detected during the study, which may be due to the fact that the virus was reported to be more common among individuals with history of haemophilia or injection drug users. Similarly, vertical and prenatal transmission of HCV is low and sexual transmission was found to less common

\section{CONCLUSION}

From the results obtained from the research, it can be concluded that HBV is highly endemic among HIV infected patients in Kano and the males are more prone to the viral infection than the females and also age group 31 - 40 were more prone to the infection. Similarly, it can also be concluded that HCV, coinfection is rare among the HIV infected individuals in Kano. It can be recommended that HIV infected individuals should be routinely subjected to HBSAg screening in order to make HIV treatment more effective. Similarly, efforts should be intensified in order to prevent HCV infection among HIV negative and HIV positive individuals in the community which can be achieved through vaccination/immunization and public enlightenment. 


\section{REFERENCES}

Alter, M.J. (2006) Epidemiology of Viral Hepatitis and HIV Co - Infection. J. Hepatology 446 - 9.

Cheesbrough, C. (2004): District Laboratory Practice in Tropical Countries. Part 2, Cambridge University Press, Cambridge. Pp $250-251$

Drosten, C, Nippraschk, T, Gamegold, C., Meisel, H., Broxne, V., Roth, W.K. Apedjimou, A, and Gunthers (2004): Prevalence of Hepatitis B virus DNA in anti - HBC positive HBSAg negative Sera correlates with $\mathrm{HCV}$ but not HIV serostatus J. of clinical virology, 2959 68.

FMH, 2008. UNFPA Priority Areas of Support and Key Achievements/Contributions to Nigeria's HIV/AIDS Response. www.fmh.gov.ng/nada3/index assessed on $8^{\text {th }}$ May, 2014.

Federal Ministry of Health of Nigeria (2003) National HIV/AIDS and Reproductive Health Survey, Abuja, Pp. 209

Finlayson, M.D.C, Hayes, P.C. and Simpson, K.J. (1999). Diseases of the Liver and Blood systems. Hepatitis, C. Haslett, Chilvery, J.A.A., Hunter.

Hodges, M., Sanders, E. and Aitken, C. 1998. Seroprevalence of hepatitis markers; HAV, HBV and HCV amongst primary school children in Freetown, Sierra Leone. W. Afr. J. Med. 17;3-7

Merchante, N, Giron - Gonzale Z, J.A., and Gonzalez S.M. (2006) Survival and Prognostic Factors for HIV infected patients with HCV related end stage Liver Diseases. AIDS 2049 - 57

Pineda, J.A, and Macais, J. (2005): Progression of Liver Fibrosis in Patients Co - Infection with HCV and HIV Undergoing antiretroviral Therapy. Journal of Anti Microbial Chemotherapy 5: $417-419$

Seef, L.B (1998): Natural History of Hepatitis C. Am J. Med. $10710-15$
Taura, W.D (2007) Studies on the Epidemiology and Chemical Expression of HBV and HCV viral Infection in Hospital Communities in Kano State. PhD. Thesis unpublished submitted to the Department of Biological Sciences, Bayero University, Kano.

Thio, C.L. (2003): Hepatitis B in HIV infected patients. Epidemiology, Natural History and Treatment. Semin Liver Dis., 23: 125 - 136.

W.H.O, (2002). Global Health Sector Strategy for HIV/AIDS General. Pp.32

Zeore, B. and Walter, B (1990): Evolution Biology Encyclopedia of Science and Technology Departments of Biology Science, Columbia University. Vol. 6, Pp. 1090 -1092. 